\title{
Community Psychological And Behavioural Responses To Coronavirus Disease 2019 Over One Year Of The Pandemic In 2020 In Hong Kong
}

\section{Qiuyan Liao}

The University of Hong Kong, Hong Kong Special Administrative Region Jingyi Xiao

The University of Hong Kong, Hong Kong Special Administrative Region Justin Cheung

The University of Hong Kong, Hong Kong Special Administrative Region

\section{Tiffany W. Y. Ng}

The University of Hong Kong, Hong Kong Special Administrative Region

\section{Wendy W. T. Lam}

The University of Hong Kong, Hong Kong Special Administrative Region

\section{Michael Y. Ni}

The University of Hong Kong, Hong Kong Special Administrative Region

\section{Benjamin J Cowling ( $\nabla$ bcowling@hku.hk)}

The University of Hong Kong, Hong Kong Special Administrative Region

\section{Research Article}

Keywords: psychological distress, risk perception, precautionary behaviours, pandemic

Posted Date: June 7th, 2021

DOl: https://doi.org/10.21203/rs.3.rs-512581/v1

License: (c) (i) This work is licensed under a Creative Commons Attribution 4.0 International License.

Read Full License

Version of Record: A version of this preprint was published at Scientific Reports on November 18th, 2021. See the published version at https://doi.org/10.1038/s41598-021-00616-9. 


\section{Abstract}

Background: Monitoring community psychological and behavioural responses to coronavirus disease 2019 (COVID-19) is important for informing policy making and risk communication to sustain public compliance with challenging precautionary behaviours and mitigating the psychological impacts.

Methods: Monthly telephone surveys in January-April 2020 and then weekly surveys from May through December 2020 were conducted to monitor changes in public risk perception of COVID-19, personal efficacy in self-protection, confidence in the government's ability to control the pandemic, precautionary behaviours, perceived impact of precautionary behaviours, psychological fatigue and distress in Hong Kong, and examine their inter-relationships.

Results: While worry about contracting COVID-19 increased, personal efficacy and confidence in the government declined as the community incidence of COVID-19 increased. The public maintained high compliance with most precautionary behaviours throughout but relaxed behaviours that were more challenging when disease incidence declined. Public confidence in the government was persistently low throughout, of which, a lower level was associated with more psychological fatigue, lower compliance with precautionary behaviours and greater psychological distress. Perceived greater negative impact of precautionary behaviours was also associated with more psychological fatigue which in turn was associated with relaxation of precautionary behaviours. Female, younger and unemployed individuals reported greater psychological distress throughout different stages of the pandemic.

Conclusions: Risk communication should focus on promoting confidence in self-protection and pandemic control to avoid helplessness to act when the pandemic resurges. Policy making should prioritize building public trust, enhancing support for sustaining precautionary behaviours, and helping vulnerable groups to adapt to the stress during the pandemic.

\section{Highlights}

- Confidence in government was low throughout the pandemic in Hong Kong.

- Lower confidence in government was associated more psychological fatigue.

- Psychological fatigue contributed to relaxation of precautionary behaviours.

- Lower confidence in government was associated with more psychological distress.

- Unemployed individuals had greater psychological distress throughout the pandemic.

\section{Implications}

Practice: Risk communication should focus on promoting the public's confidence in self-protection and pandemic control as the pandemic resurges to avoid hopelessness to act and providing appropriate feedbacks for public precautionary behaviours to avoid psychological fatigue. 
Policy: Policy making should prioritize building public trust, enhancing support for sustaining precautionary behaviours, and helping vulnerable groups to adapt to the stress during the pandemic.

Research: Future research can study what feedback messages should be provided for the public to mitigate psychological fatigue and sustain challenging precautionary behaviours.

\section{Introduction}

The coronavirus disease 2019 (COVID-19) pandemic has caused enormous global disruption. Hong Kong with population 7.5 million had reported 11,461 cases of COVID-19 including 203 fatal cases as of 30 March 2021 (Hong Kong Center for Health Protection, 19 March 2021). Although the number of COVID-19 cases to date has been relatively small, Hong Kong had experienced a number of surges in transmission in 2020 (Hong Kong Center for Health Protection, 19 March 2021). Prior to the availability of an effective vaccine, public engagement with public health and social measures (PHSMs) was critical for pandemic control (Cowling et al., 2020). Public perception of their vulnerability to and the severity of the disease, personal confidence in self-protection and trust in the government in pandemic control are important determinants of compliance with PHSMs during a pandemic (Bish \& Michie, 2010; Leung et al., 2003; Liao, Cowling, Lam, Ng, \& Fielding, 2010; Liao, Cowling, Lam, \& Fielding, 2011; Storopoli, Braga da Silva Neto, \& Mesch, 2020). According to the Transactional Theory of Stress and Coping (TTSC) (Lzarus \& Folkman, 1984), the primary stressor (e.g. COVID-19) stimulates stress appraisal which comprises evaluation of potential threat, harm caused by the stressor and individual resources or capacity to manage the stress. The stress appraisal subsequently motivates various behavioural coping strategies. TTSC also proposes that appraisal of the primary stressor (i.e., the pandemic) and the secondary stressors (e.g., the control measures) could cause enormous psychological impact (Lzarus \& Folkman, 1984). This is evidenced in the current pandemic. For instance, studies had reported a prevalence of $35 \%$ of psychological distress in Chinese adults (Qiu et al., 2020), a prevalence of around $15 \%, 8 \%$ and $5 \%$ of moderate, moderately severe and severe depression symptoms, respectively, in US adults (Ettman et al., 2020), and a prevalence of $27 \%$ of mental distress of clinical significance in UK adults (Pierce et al., 2020). Stress results from the interaction between personal resource (internal and external) and the situation (McLeod, 2012). The prolonged PHSMs may disproportionally affect and cause more psychological distress in the population who have fewer resources or perceive lower personal capacity to cope with the changing situation such as females, younger adults and people of lower income, with lower job security and being unemployed (González-Sanguino et al., 2020; Qiu et al., 2020; Xiong et al., 2020). Most studies on public psychobehavioural responses to COVID-19 were conducted during the initial stage of the pandemic or when the countries were lockdown (Ettman et al., 2020; González-Sanguino et al., 2020; Luo, Guo, Yu, Jiang, \& Wang, 2020; Pierce et al., 2020; Qiu et al., 2020; Salari et al., 2020; Storopoli et al., 2020). There remained limited understanding about the psychological impact in the general public throughout different stages of the pandemic. In addition, according to Model of Self-regulation (MSR) (Carver, Scheier, \& Fulford, 2008), the process of coping with stress can also be viewed as a selfregulatory process through which individuals take actions to cope with the changing situation to achieve certain personal goals (e.g. self-protection or pandemic control). This subsequently generates a feedback 
process through which individuals evaluate whether their personal goals are achieved through the effort input. If they constantly receive negative feedbacks (e.g., the pandemic repeatedly surges and negative impacts of the coping strategies), it will cause feeling of self-regulation failure and fatigue (e.g., pandemic fatigue) (Michie, West, \& Harvey, 2020; World Health Organization, 2020) which will in turn demotivates existing coping effort (e.g., compliance with precautionary behaviours). How individuals appraised their precautionary behaviours (particularly physical distancing behaviours) would affect precautionary behaviours against the COVID-19 pandemic was seldom studied.

This was a repeated cross-sectional study to monitor the changes of public stress appraisal including COVID-19 risk perception, personal efficacy and confidence in government's pandemic control, behavioural coping (i.e., precautionary behaviours) and psychological distress over one year of the pandemic in 2020 in Hong Kong. We also aimed to examine the interrelationships among appraisal of stress and precautionary behaviours, adoption of precautionary behaviours and psychological distress, and to identify socioeconomic strata associated with increased vulnerability to psychological distress throughout different stages of the pandemic.

\section{Materials And Methods}

\section{Study design and sampling}

Repeated cross-sectional surveys were conducted from January 2020, immediately before any cases of COVID-19 were reported in Hong Kong, through to December 2020. Surveys were conducted monthly from January to April 2020 with a sample size of approximately 1000 in each round, and survey frequency increased to weekly thereafter with the sample size alternating between 500 and 1000 in each round. Adults (aged $\geq 18$ years) were recruited using random digit dialling of landline and mobile phones with a ratio of 1:1 randomly generated by computer. Participants were included if they could speak Cantonese and were capable of answering a telephone interview. One adult within each selected household whose birthday was soonest for residential phone calls or the owners of the mobile phone numbers for mobile phone calls were invited to complete the telephone interview. Calls were made during non-working hours as well as working hours to avoid oversampling of non-working groups. Participants were newly recruited in each round. Each round was conducted for a period of 3-5 days and each interview was kept within 15 minutes. Informed consent was obtained from all participants. This study was conducted in accordance with the Declaration of Helsinki and Good Clinical Practices. And the study received ethical approval from the Institutional Review Board of the University of Hong Kong.

\section{Study instruments}

The questionnaire for each survey round included the same core study measures throughout, with additional measures included in a subset of survey weeks to ensure feasible length of each questionnaire while enabling examination of a wide spectrum of influences on stress appraisal, precautionary behaviours and mental health outcomes. Core study measures included perceived personal susceptibility from COVID-19, worry about contacting COVID-19, perceived severity of COVID-19, personal efficacy in 
self-protection, confidence in the government's ability to control the pandemic, personal hygiene practices (PHP), physical distancing behaviours (PDB) and psychological distress. Measures of COVID-19-related risk perceptions, beliefs and precautionary behaviours were similar to that used in our surveys during the outbreak of severe respiratory syndrome (SARS) in 2003 (Leung et al., 2005), influenza A(H1N1) pandemic in 2009-10 (Cowling et al., 2010; Liao, Cowling, Lam, Ng, \& Fielding, 2014) and outbreaks of avian influenza A(H7N9) in 2013-15 (Liao, Wu, Wing Tak Lam, Cowling, \& Fielding, 2019). Psychological distress was assessed with the 4-item Patient Health Questionnaire (PHQ-4) that comprises two items from the Generalized Anxiety Disorder-7 (GAD-7) and two items from the PHQ-9 depression measure (Kroenke, Spitzer, Williams, \& Löwe, 2009; Löwe et al., 2010). Each item of the PHQ-4 was rated on a Likert scale ranging from 0 ('not at all') to 3 ('nearly every day') with a higher score indicating greater distress. A summed scores of $\geq 3$ of the two items from GAD-7 was used for assessing probable anxiety (Plummer, Manea, Trepel, \& McMillan, 2016) while that of the two items from PHQ-9 was used for assessing probable depression (Staples et al., 2019). The total scores of all four items of PHQ-4 were also calculated to indicate overall psychological distress (Kroenke et al., 2009; Löwe et al., 2010). In Round 25 and Round 33, perceived negative impacts of physical distancing measures and 'psychological fatigue' with the pandemic were measured. According to the World Health Organization (WHO), psychological fatigue with the pandemic is defined as feeling of distress or frustration due to "sustained and unresolved adversity" (World Health Organization, 2020). Based on this definition, psychological fatigue was operationalized as feeling of tiredness with COVID-19 and emotional exhaustion (helplessness and frustration) regarding pandemic control in this study. Detailed descriptions of the study measures were provided in Appendix Table 1.

Table 1. Associations of probable anxiety and probable depression with socio-demographics and phases of pandemic 


\begin{tabular}{|c|c|c|}
\hline & \multicolumn{2}{|c|}{ Odds ratio ( $95 \%$ confidence interval) } \\
\hline & $\begin{array}{l}\text { Probable } \\
\text { anxiety }\end{array}$ & $\begin{array}{l}\text { Probable } \\
\text { depression }\end{array}$ \\
\hline Sex (male vs. female) & $\begin{array}{l}0.86(0.79- \\
0.94)^{b}\end{array}$ & $1.02(0.93-1.13)$ \\
\hline \multicolumn{3}{|l|}{ Age (years) } \\
\hline $18-24$ & 1.00 & 1.00 \\
\hline $25-34$ & $1.14(0.94-1.39)$ & $1.16(0.95-1.41)$ \\
\hline $35-44$ & $1.04(0.85-1.27)$ & $0.86(0.69-1.06)$ \\
\hline $45-54$ & $\begin{array}{l}0.78(0.63- \\
0.96)^{\mathrm{a}}\end{array}$ & $0.60(0.48-0.74)^{c}$ \\
\hline $55-64$ & $\begin{array}{l}0.67(0.54- \\
0.83)^{\mathrm{c}}\end{array}$ & $0.55(0.44-0.69)^{\mathrm{c}}$ \\
\hline$\geq 65$ & $\begin{array}{l}0.58(0.45- \\
0.74)^{\mathrm{c}}\end{array}$ & $0.51(0.39-0.66)^{\mathrm{c}}$ \\
\hline \multicolumn{3}{|l|}{ Educational attainment } \\
\hline Primary or below & 1.00 & 1.00 \\
\hline Secondary & $\begin{array}{l}0.77(0.67- \\
0.88)^{\mathrm{c}}\end{array}$ & $0.89(0.76-1.04)$ \\
\hline Tertiary or above & $\begin{array}{l}0.60(0.51- \\
0.71)^{c}\end{array}$ & $0.69(0.57-0.82)^{\mathrm{c}}$ \\
\hline \multicolumn{3}{|l|}{ Occupation } \\
\hline Executive and professional & 1.00 & 1.00 \\
\hline Clerical and service worker & $1.00(0.89-1.13)$ & $1.07(0.93-1.23)$ \\
\hline Production worker & $1.06(0.90-1.25)$ & $1.09(0.91-1.31)$ \\
\hline Student & $1.10(0.87-1.39)$ & $1.10(0.86-1.42)$ \\
\hline Homemaker/housewife & $1.10(0.94-1.29)$ & $1.15(0.96-1.38)$ \\
\hline Retirement & $1.04(0.87-1.25)$ & $1.26(0.92-1.38)$ \\
\hline Unemployed or seeking jobs & $\begin{array}{l}1.84(1.54- \\
2.20)^{\mathrm{c}}\end{array}$ & $2.09(1.72-2.54)^{\mathrm{C}}$ \\
\hline \multicolumn{3}{|l|}{ Epidemic phase } \\
\hline Phase 1 (initial phase with mainly imported cases) & 1.00 & - \\
\hline
\end{tabular}




\begin{tabular}{|c|c|c|}
\hline Phase 2 (relative quiescent phase) & $\begin{array}{l}0.74(0.65- \\
0.85)^{\mathrm{c}}\end{array}$ & 1.00 \\
\hline Phase 3 (first major community epidemic) & $0.88(0.77-1.00)$ & $1.10(0.97-1.24)$ \\
\hline $\begin{array}{l}\text { Phase } 4 \text { (phase with relatively low community } \\
\text { incidence) }\end{array}$ & $\begin{array}{l}0.62(0.54- \\
0.70)^{\mathrm{C}}\end{array}$ & $0.82(0.73-0.92)^{b}$ \\
\hline Phase 5 (second major community epidemic) & $\begin{array}{l}0.75(0.65- \\
0.86)^{\mathrm{c}}\end{array}$ & $0.95(0.83-1.09)$ \\
\hline
\end{tabular}

${ }^{a} p<0.05,{ }^{b} p<0.01,{ }^{c} p<0.001$

\section{Data analysis}

The analytic strategies involved three stages. First, means or proportions of core study measures were calculated and weighted to the Hong Kong census data on age and sex in 2019 and plotted by survey round.

Second, to ensure sufficient sample sizes for the comparisons of mental health outcomes by sociodemographic strata, survey rounds were combined to represent five phases of the COVID-19 epidemic in Hong Kong in 2020: Phase 1 (January-April (Round 1-4))-an early phase of the epidemic characterized by mainly cases imported from mainland China and overseas, Phase 2 (May-June (Round 5-12)) -a quiescent phase characterized by merely sporadic cases, Phase 3 (July-August (Round 1320)) -the first major community epidemic of COVID-19 characterized by widespread local-acquired infections, Phase 4 (August-November (Round 21-33)) when community incidence was lower again and Phase 5 (November-December (Round 34-39)) -the second major community epidemic of COVID-19. Weighted proportions of probable anxiety and probable depression by major sociodemographic strata were compared and plotted by the five phases. Logit regression enabled examination of associations of probable anxiety and probable depression with major sociodemographic factors adjusting for epidemic phase.

The third stage involved testing two structural equation models (SEM) based on TTSC (Lzarus \& Folkman, 1984) and MSR (Carver et al., 2008) to understand public stress appraisal, coping and the selfregulation process during the pandemic. Model 1 was a basic model in which stress appraisal involving COVID-19 risk perceptions, personal efficacy and confidence in the government were hypothesized to influence PHP, PDB and psychological distress. Socio-demographic factors including age, sex, education attainment and occupation status that indicated resources and capacity to cope with stress were hypothesized to be associated with stress appraisal in this model. Model 2 was aimed to test a feedback process of the self-regulatory effort. We hypothesized that negative reappraisal of personal effort (for achieving personal goals) including reduced personal efficacy, reduced confidence in the government and perceived more negative impacts of the physical distancing measures could lead to a feeling of selfregulatory failure and thereby more feeling of fatigue and greater psychological distress. We also hypothesized that the feeling of fatigue due to negative appraisal of their self-regulatory effort could in 
turn reduce compliance with precautionary behaviours. Multiple-item measures were treated as latent variables for which confirmatory factor analysis (CFA) was first run to test their correlations before they were included into the full SEM. Robust maximum likelihood estimator was used to estimate the SEM models. Multiple model fit indices including $\mathrm{CFI}$, TLI, SRMR and RMSEA were used to assess model fit with $\mathrm{CFI}>0.90, \mathrm{TLI}>0.90$, SRMR $<0.08$ and RMSEA $<0.08$ indicating a good fit of the model to the data.

The CFA and SEM were running using Mplus 7.3 for Windows which enables handling missing data using full information maximum likelihood.

\section{Results}

\section{Participants}

A total of 39 rounds of telephone surveys were conducted from January to December 2020. Overall, a total of 32,439 subjects answered the calls and were screened to be eligible, of whom, $30,827(95.0 \%)$ completed the interviews (Fig. 1).

\section{Temporal changes in stress appraisal, precautionary behaviours and psychological distress}

Figure 2 shows the temporal changes in stress appraisal, precautionary behaviours and psychological distress. Prevalence of some stress appraisal and precautionary behaviours measures in Round 1-3 had been previously reported (Cowling et al., 2020) but were also included here for comparison. The prevalence of perceiving vulnerable to COVID-19 were relatively higher in the first two rounds (17-18\%) but declined to around $10-15 \%$ throughout with only slight increases when the community incidence of COVID-19 was higher (Panel A). Prevalence of having at least moderate worry about contacting COVID-19 was around $30-40 \%$ when the disease incidence was low but increased to more than $60 \%$ when the disease incidence reached a peak (Panel A). Around 90\% perceiving the severity of COVID-19 to be severe or very severe initially, which declined to $50-60 \%$ in late 2020 except for a slight increase in December 2020 when there was a small bounce in number of death cases due to COVID-19 (Panel A). Around 60$70 \%$ believing in personal ability in the prevention against COVID-19 when the disease incidence was low, which declined to $50 \%$ when locally acquired cases increased rapidly in the community (Panel B). However, having Confidence in the Government was low, being 30-50\% throughout and declined to below $30 \%$ when disease incidence increased (Panel B).

Prevalence of avoiding crowded places, avoiding social gathering and maintaining appropriate social distance with others outside of family were maintained at a level of more than $70-80 \%$ throughout and reached to more than $90 \%$ when disease incidence was high (Panel C). Avoiding going out, avoiding healthcare facilities and avoiding non-essential use of public transport (e.g., for leisure activity) were seen to decline more when disease incidence was lower but increased to $70-80 \%$ or more when disease incidence was higher (Panel C).

The prevalence of wearing facemasks when going outside was almost $100 \%$ since Round 2 when COVID19 cases started to report in Hong Kong (Panel D). Prevalence of washing hands after going outside increased dramatically within the first four months of the pandemic and was maintained at a level of 
more than $90 \%$ since Round 5 (Panel D). Around $80 \%$ and $70 \%$ reported using liquid soap and rubbing hands with soap for at least 20 second, respectively, when washing hands throughout (Panel D). Around $50 \%-60 \%$ reported avoiding touching common objects in public places but $70 \%-80 \%$ would wash or disinfect hands immediately after touching public objects (Panel D).

Probable anxiety reached a peak of $\sim 20 \%$ when COVID-19 cases were first reported in Hong Kong which declined thereafter to around 10\% with additional peaks detected in Round 6, Round 18 and Round 38, respectively, when community incidence increased or social distancing measures were prolonged even if community incidence substantially declined (Panel E). The temporal trends of probable depression and overall psychological distress score were similar (Panel E).

\section{Psychological distress by socio-demographic strata}

Females were more likely to have probable anxiety but not probable depression while younger, less educated and unemployed participants were more likely to have both probable anxiety and probable depression than their counterparts (Table 1). Throughout the five phases of the pandemic, the prevalence of probable anxiety was consistently higher in females than males, in participants aged 18-24 years and 25-44 years than those who were older and in participants who were unemployed or seeking jobs (Fig. 3). Participants aged 18-24 years and 25-44 years and those who were unemployed or seeking jobs were also found to have a higher prevalence of probable depression throughout the five phases of the pandemic (Fig. 3).

\section{Stress appraisal, precautionary behaviours, psychological distress and the self-regulation process}

In Model 1 (Panel A, Fig. 4), we found that higher personal efficacy $(\beta=-0.38, S E=0.009, p<0.001)$ and confidence in the government $(\beta=-0.18$, SE $=0.004 p<0.001)$ was associated with lower risk perception of COVID-19 while higher risk perception of COVID-19 was associated with more adoption of PHP $(\beta=0.28$, $\mathrm{SE}=0.006, p<0.001)$, more PDB $(\beta=0.32, \mathrm{SE}=0.004, p<0.001)$ and greater psychological distress $(\beta=$ $0.34, \mathrm{SE}=0.012, p<0.001)$. Personal efficacy was positively associated with adoption of PHP $(\beta=0.18$, $\mathrm{SE}=0.003, p<0.001)$ and $\mathrm{PDB}(\beta=0.12, \mathrm{SE}=0.002, p<0.001)$ while more confidence in the government was associated with more PDB $(\beta=0.13, \mathrm{SE}=0.001, p<0.001)$ and less psychological distress $(\beta=-0.12$, $\mathrm{SE}=0.004, p<0.001)$. In addition, we found that older individuals perceived lower risk from COVID-19 $(\beta=-0.10, \mathrm{SE}=0.003, p<0.001)$ and had more confidence in government's pandemic control $(\beta=0.31, \mathrm{SE}$ $=0.004, p<0.001)$ while better educated individuals had lower confidence in government $(\beta=-0.10, \mathrm{SE}=$ $0.017, p<0.001$ ). In Model 2 (Panel B, Fig. 4), we found that more negative appraisal of physical distancing measures was associated with greater psychological distress $(\beta=0.39, \mathrm{SE}=0.029, p<0.001)$, and greater psychological fatigue $(\beta=0.75, \mathrm{SE}=0.040, p<0.001)$. Lower confidence in the government was also associated with greater psychological fatigue $(\beta=-0.30, S E=0.010, p<0.001)$ which in turn was associated with lower adoption of PHP $(\beta=-0.13, \mathrm{SE}=0.026, p=0.001)$ and $\operatorname{PDB}(\beta=-0.29, \mathrm{SE}=0.037, p<$ 0.001). Details of the SEM results are provided in Appendix Table 2 and Appendix Table 3.

\section{Discussion}


Our study showed that public worry about contracting COVID-19 changed in line with the community incidence of COVID-19 throughout the first year of the pandemic. Perceived susceptibility to COVID-19 was initially higher but declined thereafter and maintain at a low level. The discrepancy between worry about and perceived susceptibility to COVID-19 suggests that emotional response to a threat is more context- and value-driven rather than a mere reflection of cognitive estimate of probability of infection (Sjöberg, 1998). Emotional response to a hazard can be more heuristic and quicker to help people navigate during uncertain and complex situations while cognitive judgement of risk probability tends to be subject to biases (Sjoberg, 2000; Slovic, Finucane, Peters, \& MacGregor, 2004). Both personal efficacy in self-protection and confidence in the government's ability to control the pandemic significantly declined when widespread of COVID-19 in the community was seen. This indicates that public precautionary behaviours tend to be worry-driven and if low confidence in self-prevention and pandemic control was not appropriately addressed could lead to helplessness to act (Witte \& Allen, 2000). In the context of a preexisting low level of public trust in the government in Hong Kong relating to major social unrest since June 2009 ( $\mathrm{Ni}$ et al., 2020) and the historical experience of SARS in 2003-2004 (Lee, 2009), public trust in the government appeared to quickly erode and difficult to recover, posing enormous challenges for the Hong Kong government for managing the pandemic.

While 'behavioural fatigue', one's declining ability to perform behaviours over time due to complex underlying psychological mechanism, is questioned to be real (Harvey, 2020), the phenomenon of 'pandemic fatigue' is documented by the World Health Organization (WHO) (World Health Organization, 2020). Our current study found that the population were able to maintain high compliance with most precautionary behaviours such as good hand hygiene, avoiding crowded places and avoiding social gathering throughout the first year of the pandemic but behaviours such as avoiding going outside, avoiding healthcare facilities and avoiding non-essential use of public transport (e.g., for holidays) tend to relax when community incidence of COVID-19 declined. This indicates that the relaxation of some precautionary behaviours is not the result of the impaired ability to perform the tasks over time but the declining worry about COVID-19 and possibly the high cost of sustaining these behaviours (Michie et al., 2020). However, we found that negative appraisal of personal effort and its failure to achieve certain goals (pandemic control) as well as negative appraisal of the coping strategies (i.e., physical distancing measures) did associate with feeling of fatigue which in turn was associated with lower compliance with the precautionary behaviours. This highlights the importance of a self-regulatory feedback process to sustain compliance with challenging behaviours. Lacking confidence in the government's control measures may further deteriorate the feeling of fatigue. During a crisis, a trustworthy government can help set a clear reward or punishment system to provide feedbacks for the public's precautionary behaviours (Bavel et al., 2020). Lacking such feedbacks for the great efforts put on compliance with the challenging behaviours could increase the feeling of 'fatigue'.

The prevalence of probable anxiety and probable depression throughout the first year of the pandemic in Hong Kong was lower than the synthesized prevalence of anxiety and depression reported by recent systematic reviews (Luo et al., 2020; Salari et al., 2020). This indicates that the pandemic may not have resulted in relatively higher substantial distress in the population in Hong Kong. However, disparities in 
the psychological impacts of the pandemic across socio-demographic strata were found in our study. Similar to others' study (Bräscher et al., 2021; González-Sanguino et al., 2020; Pierce et al., 2020; Qiu et al., 2020), female, younger, less educated and unemployed individuals were more vulnerable to psychological distress. The interaction between the environment and individuals' resources to cope with was more important than the situation itself for individuals' stress response (McLeod, 2012). Individuals who perceive more personal and the government's capacity to cope with the threat could shape their meaning of the situation which in turn determines their psychological distress. Confidence in the government and believing that they have done what is needed for the best interest of public health are important to determine to what extent the public would tolerate and accept the challenging control measures (Storopoli et al., 2020) and hence also has a direct psychological impact. We found that female and less educated individuals perceived more risk from/of COVID-19 while younger and less educated individuals perceived lower personal efficacy. Younger individuals also had least confidence in the government. This may explain why these socio-demographic groups reported more anxiety and/or depression. Unemployment was not associated with risk perception of COVID-19, personal efficacy and confidence in the government but was persistently associated with greater anxiety and depression throughout the pandemic. Possible reasons may be that unemployment impair not only financial capacity to cope with the stress but also sense of self-esteem, personal identity and social connections (Achdut \& Refaeli, 2020; Blustein \& Guarino, 2020; Mimoun, Ari, \& Margalit, 2020).

Our study had some limitations. First, the cross-sectional nature of our data means that we are not able to examine the within-person changes of the psycho-behavioural responses throughout the pandemic and that the associations examined in our study may be attenuated by reverse causality. Second, sampling errors may generate differences in major study outcomes across survey rounds though each round had a relatively large sample size and all study outcomes were weighted by population age and sex. Third, some of our study constructs were assessed using single items, which means that these constructs may not be adequately assessed and thereby measurement errors may affect the reliability of parameter estimates. However, there are concerns over response rates and survey efficiency if long questionnaires were used.

\section{Conclusions}

Our study suggests that risk communication should focus on promoting the public's confidence in selfprotection and pandemic control as the pandemic resurges to avoid defensive response and feeling hopeless to act (Witte \& Allen, 2000). Rebuilding public trust in the government should be a political priority to reduce psychological fatigue and psychological distress and sustain compliance with the precautionary behaviours. The government and relevant organizations should also provide practical support for the public to sustain adherence to more challenging behaviours rather than blaming the nonadherence (Michie et al., 2020) and proper positive feedbacks and encouragement of their precautionary behaviours to reduce psychological fatigue during a long-lasting catastrophe (Bonell et al., 2020). Furthermore, our study suggests that more social support should be provided for females, younger, less educated and unemployed individuals to help them adapt to the stress caused by the pandemic. In 
particular, enhancing job security, preventing job loss and creating opportunity for re-employment should be put as one priority of policy making during the pandemic.

\section{Declarations}

\section{Financial support}

This study was supported by funding from the Government of the Hong Kong Special Administrative Region, the Health and Medical Research Fund of the Food and Health Bureau of the Hong Kong SAR Government (Project No. COVID19F04).

\section{Conflicts of Interest}

BJC consults for Roche, Sanofi Pasteur, GSK and Moderna. The authors report no other potential conflicts of interest.

\section{Author's Contribution}

Designed study: QL, BJC, WWTL and MYN. Co-ordinated data collection: JX and TWYN. Analysed data: QL, JX, JC and BJC. Wrote first draft of manuscript: QL. Contributed to manuscript revision and approved final draft: All authors.

\section{References}

1. Achdut, N., \& Refaeli, T. (2020). Unemployment and Psychological Distress among Young People during the COVID-19 Pandemic: Psychological Resources and Risk Factors. International Journal of Environmental Research and Public Health, 17(19), 7163. doi:10.3390/ijerph17197163

2. Bavel, J. J. V., Baicker, K., Boggio, P. S., Capraro, V., Cichocka, A., Cikara, M.,.. . Willer, R. (2020). Using social and behavioural science to support COVID-19 pandemic response. Nature Human Behaviour, 4(5), 460-471. doi:10.1038/s41562-020-0884-z

3. Bish, A., \& Michie, S. (2010). Demographic and attitudinal determinants of protective behaviours during a pandemic: A review. British Journal of Health Psychology, 15(Pt 4), 797-824. doi:10.1348/135910710X485826

4. Blustein, D. L., \& Guarino, P. A. (2020). Work and Unemployment in the Time of COVID-19: The Existential Experience of Loss and Fear. Journal of Humanistic Psychology, 60(5), 702-709. doi:10.1177/0022167820934229

5. Bonell, C., Michie, S., Reicher, S., West, R., Bear, L., Yardley, L.,.. . Rubin, G. J. (2020). Harnessing behavioural science in public health campaigns to maintain 'social distancing' in response to the 
COVID-19 pandemic: key principles. Journal of Epidemiology and Community Health, 74(8), 617619. doi:10.1136/jech-2020-214290

6. Bräscher, A. K., Benke, C., Weismüller, B. M., Asselmann, E., Skoda, E. M., Teufel, M. A.,.. . Pané-Farré, C. A. (2021). Anxiety and depression during the first wave of COVID-19 in Germany - results of repeated cross-sectional surveys. Psychological Medicine, 1-10. doi:10.1017/S0033291721000866

7. Carver, C. S., Scheier, M. F., \& Fulford, D. (2008). Self-regulatory processes, stress, and coping. In Handbook of personality: Theory and research, 3rd ed. (pp. 725-742). New York, NY, US: The Guilford Press.

8. Cowling, B. J., Ali, S. T., Ng, T. W. Y., Tsang, T. K., Li, J. C. M., Fong, M. W.,.. . Leung, G. M. (2020). Impact assessment of non-pharmaceutical interventions against coronavirus disease 2019 and influenza in Hong Kong: an observational study. The Lancet Public Health, 5(5), e279-e288. doi:10.1016/S2468-2667(20)30090-6

9. Cowling, B. J., Ng, D. M., Ip, D. K., Liao, Q., Lam, W. W., Wu, J. T.,.. . Fielding, R. (2010). Community psychological and behavioral responses through the first wave of the 2009 influenza $A(H 1 N 1)$ pandemic in Hong Kong. Journal of Infectious Diseases, 202(6), 867-876. doi:10.1086/655811

10. Ettman, C. K., Abdalla, S. M., Cohen, G. H., Sampson, L., Vivier, P. M., \& Galea, S. (2020). Prevalence of Depression Symptoms in US Adults Before and During the COVID-19 Pandemic. JAMA Netw Open, 3(9), e2019686. doi:10.1001/jamanetworkopen.2020.19686

11. González-Sanguino, C., Ausín, B., Castellanos, M. Á., Saiz, J., López-Gómez, A., Ugidos, C., \& Muñoz, M. (2020). Mental health consequences during the initial stage of the 2020 Coronavirus pandemic (COVID-19) in Spain. Brain, Behavior, and Immunity, 87, 172-176.

doi:https://doi.org/10.1016/j.bbi.2020.05.040

12. Harvey, N. (2020). Behavioral Fatigue: Real Phenomenon, Naive Construct, or Policy Contrivance? Frontiers in Psychology, 11, 589892. doi:10.3389/fpsyg.2020.589892

13. Hong Kong Center for Health Protection. (19 March 2021). Latest situation of cases of COVID-19 (as of 30 March 2021). Retrieved from https://www.chp.gov.hk/files/pdf/local_situation_covid19_en.pdf

14. Kroenke, K., Spitzer, R. L., Williams, J. B. W., \& Löwe, B. (2009). An Ultra-Brief Screening Scale for Anxiety and Depression: The PHQ-4. Psychosomatics, 50(6), 613-621.

doi:10.1176/appi.psy.50.6.613

15. Lee, K. (2009). How the Hong Kong government lost the public trust in SARS: Insights for government communication in a health crisis. Public Relations Review, 35(1), 74-76.

doi:http://dx.doi.org/10.1016/j.pubrev.2008.06.003

16. Leung, G. M., Ho, L. M., Chan, S. K., Ho, S. Y., Bacon-Shone, J., Choy, R. Y.,.. . Fielding, R. (2005). Longitudinal assessment of community psychobehavioral responses during and after the 2003 outbreak of severe acute respiratory syndrome in Hong Kong. Clinical Infectious Diseases, 40(12), 1713-1720. doi:10.1086/429923

17. Leung, G. M., Lam, T. H., Ho, L. M., Ho, S. Y., Chan, B. H., Wong, I. O., \& Hedley, A. J. (2003). The impact of community psychological responses on outbreak control for severe acute respiratory syndrome in 
Hong Kong. Journal of Epidemiology and Community Health, 57(11), 857-863. doi:10.1136/jech.57.11.857

18. Liao, Q., Cowling, B., Lam, W. T., Ng, M. W., \& Fielding, R. (2010). Situational awareness and health protective responses to pandemic influenza A (H1N1) in Hong Kong: a cross-sectional study. PloS ONE, 5(10), e13350. doi:10.1371/journal.pone.0013350

19. Liao, Q., Cowling, B. J., Lam, W. W., \& Fielding, R. (2011). The influence of social-cognitive factors on personal hygiene practices to protect against influenzas: using modelling to compare avian A/H5N1 and 2009 pandemic A/H1N1 influenzas in Hong Kong. International Journal of Behavioral Medicine, 18(2), 93-104. doi:10.1007/s12529-010-9123-8

20. Liao, Q., Cowling, B. J., Lam, W. W., Ng, D. M., \& Fielding, R. (2014). Anxiety, worry and cognitive risk estimate in relation to protective behaviors during the 2009 influenza A/H1N1 pandemic in Hong Kong: ten cross-sectional surveys. BMC Infectious Diseases, 14(1), 169. doi:10.1186/1471-2334-14169

21. Liao, Q., Wu, P., Wing Tak Lam, W., Cowling, B. J., \& Fielding, R. (2019). Trajectories of public psychobehavioural responses relating to influenza A(H7N9) over the winter of 2014-15 in Hong Kong. Psychology \& Health, 34(2), 162-180. doi:10.1080/08870446.2018.1515436

22. Löwe, B., Wahl, I., Rose, M., Spitzer, C., Glaesmer, H., Wingenfeld, K.,.. . Brähler, E. (2010). A 4-item measure of depression and anxiety: Validation and standardization of the Patient Health Questionnaire-4 (PHQ-4) in the general population. Journal of Affective Disorders, 122(1), 86-95. doi:10.1016/j.jad.2009.06.019

23. Luo, M., Guo, L., Yu, M., Jiang, W., \& Wang, H. (2020). The psychological and mental impact of coronavirus disease 2019 (COVID-19) on medical staff and general public - A systematic review and meta-analysis. Psychiatry Research, 291, 113190. doi:10.1016/j.psychres.2020.113190

24. Lzarus, R. S., \& Folkman, S. (1984). Stress, appraisal, and coping. New York, NY: Springer Publishing Company.

25. McLeod, J. D. (2012). The Meanings of Stress: Expanding the Stress Process Model. Society and Mental Health, 2(3), 172-186. doi:10.1177/2156869312452877

26. Michie, S., West, R., \& Harvey, N. (2020). The concept of "fatigue" in tackling covid-19. British Medical Journal, 371, m4171. doi:10.1136/bmj.m4171\%J BMJ

27. Mimoun, E., Ari, A. B., \& Margalit, D. (2020). Psychological aspects of employment instability during the COVID-19 pandemic. Psychological Trauma, 12(S1), S183-S185. doi:10.1037/tra0000769

28. Ni, M. Y., Yao, X. I., Leung, K. S. M., Yau, C., Leung, C. M. C., Lun, P.,.. . Leung, G. M. (2020). Depression and post-traumatic stress during major social unrest in Hong Kong: a 10-year prospective cohort study. The Lancet, 395(10220), 273-284. doi:10.1016/S0140-6736(19)33160-5

29. Pierce, M., Hope, H., Ford, T., Hatch, S., Hotopf, M., John, A.,... Abel, K. M. (2020). Mental health before and during the COVID-19 pandemic: a longitudinal probability sample survey of the UK population. The Lancet Psychiatry, 7(10), 883-892. doi:10.1016/S2215-0366(20)30308-4 
30. Plummer, F., Manea, L., Trepel, D., \& McMillan, D. (2016). Screening for anxiety disorders with the GAD-7 and GAD-2: a systematic review and diagnostic metaanalysis. General Hospital Psychiatry, 39, 24-31. doi:10.1016/j.genhosppsych.2015.11.005

31. Qiu, J., Shen, B., Zhao, M., Wang, Z., Xie, B., \& Xu, Y. (2020). A nationwide survey of psychological distress among Chinese people in the COVID-19 epidemic: implications and policy recommendations. General Psychiatry, 33(2), e100213. doi:10.1136/gpsych-2020-100213

32. Salari, N., Hosseinian-Far, A., Jalali, R., Vaisi-Raygani, A., Rasoulpoor, S., Mohammadi, M.,.. . KhalediPaveh, B. (2020). Prevalence of stress, anxiety, depression among the general population during the COVID-19 pandemic: a systematic review and meta-analysis. Globalization and Health, 16(1), 57. doi:10.1186/s12992-020-00589-w

33. Sjoberg, L. (2000). Factors in risk perception. Risk Analysis, 20(1), 1-11. doi: https://doi.org/10.1111/0272-4332.00001

34. Sjöberg, L. (1998). Worry and Risk Perception. Risk Analysis, 18(1), 85-93. doi:https://doi.org/10.1111/j.1539-6924.1998.tb00918.x

35. Slovic, P., Finucane, M. L., Peters, E., \& MacGregor, D. G. (2004). Risk as analysis and risk as feelings: some thoughts about affect, reason, risk, and rationality. Risk Analysis, 24(2), 311-322. doi:10.1111/j.0272-4332.2004.00433.x

36. Staples, L. G., Dear, B. F., Gandy, M., Fogliati, V., Fogliati, R., Karin, E.,.. . Titov, N. (2019). Psychometric properties and clinical utility of brief measures of depression, anxiety, and general distress: The PHQ2, GAD-2, and K-6. General Hospital Psychiatry, 56, 13-18. doi:https://doi.org/10.1016/j.genhosppsych.2018.11.003

37. Storopoli, J., Braga da Silva Neto, W. L., \& Mesch, G. S. (2020). Confidence in social institutions, perceived vulnerability and the adoption of recommended protective behaviors in Brazil during the COVID-19 pandemic. Social Science \& Medicine, 265, 113477. doi:10.1016/j.socscimed.2020.113477

38. Witte, K., \& Allen, M. (2000). A Meta-Analysis of Fear Appeals: Implications for Effective Public Health Campaigns. Health Education \& Behavior, 27(5), 591-615. doi:10.1177/109019810002700506

39. World Health Organization. (2020). Pandemic fatigue reinvigarating the public to prevent COVID-19. Retrieved from https://apps.who.int/iris/bitstream/handle/10665/337574/WHO-EURO-2020-157341324-56242-eng.pdf

40. Xiong, J., Lipsitz, O., Nasri, F., Lui, L. M. W., Gill, H., Phan, L.,.. . Mclntyre, R. S. (2020). Impact of COVID19 pandemic on mental health in the general population: A systematic review. Journal of Affective Disorders, 277, 55-64. doi:10.1016/j.jad.2020.08.001

\section{Figures}




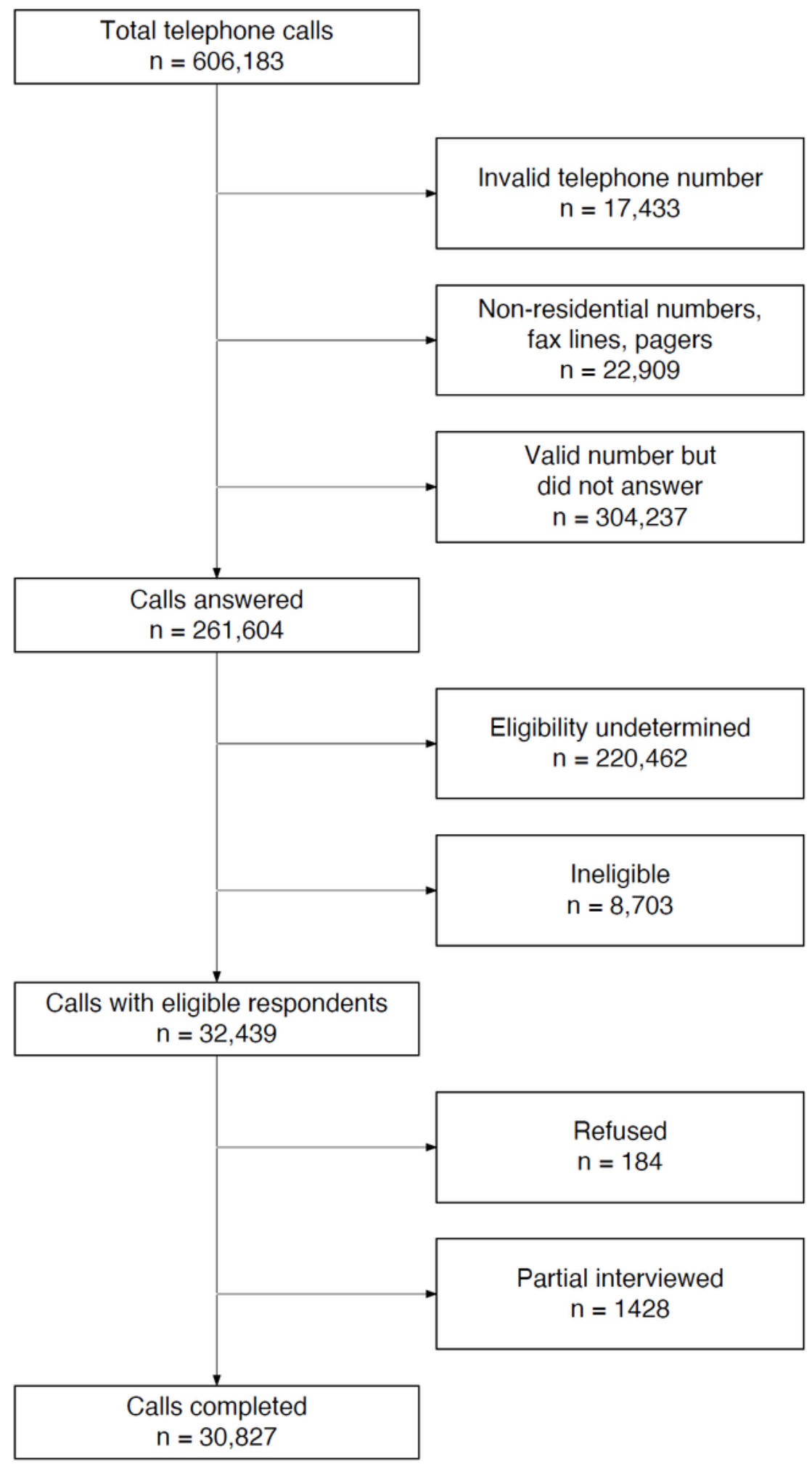

\section{Figure 1}

The flow chat of subject recruitment and interview 

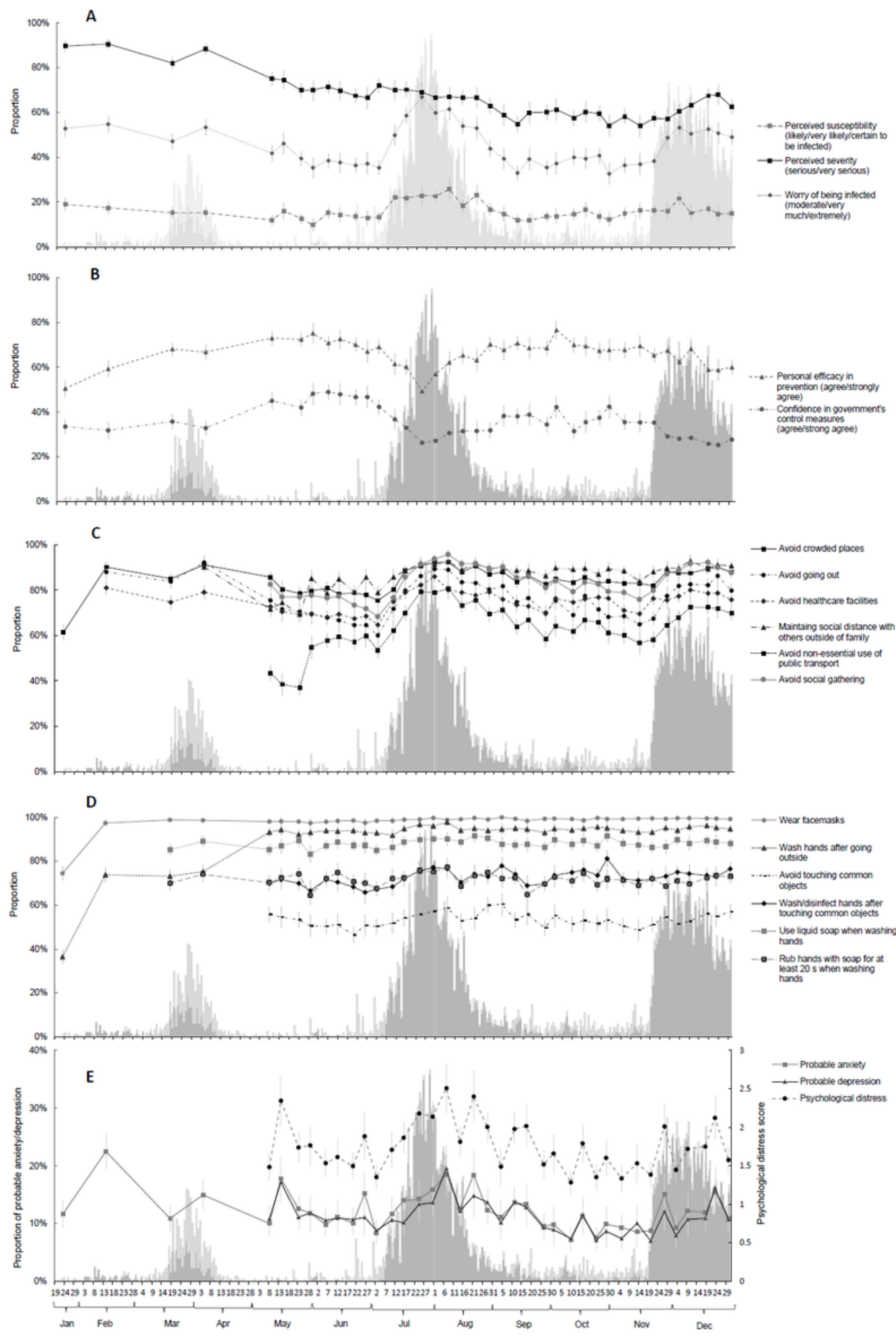

\section{Figure 2}

Daily confirmed cases of COVID-19 in Hong Kong and the temporal changes in risk perception of COVID19 (A), personal efficacy in the prevention of COVID-19 and confidence in government's control measures (B), probable anxiety, probable depression and overall psychological distress (C), adoption of physical distancing behaviours (D) and adoption of personal hygiene practices (E). 

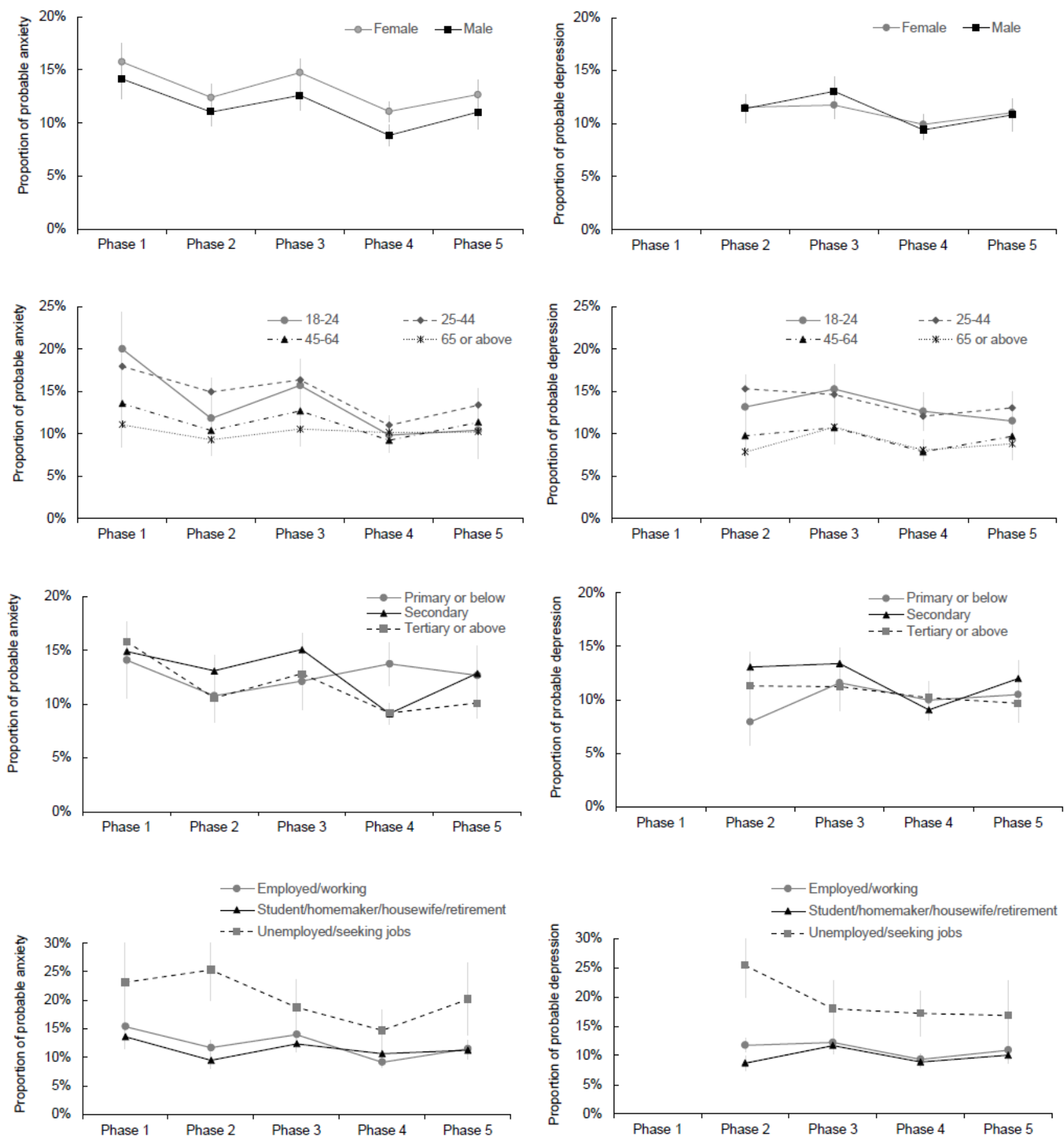

\section{Figure 3}

Proportions of respondents who had probable anxiety and probable depression by sex, age, educational attainment and occupation. 
Panel A: Model 1
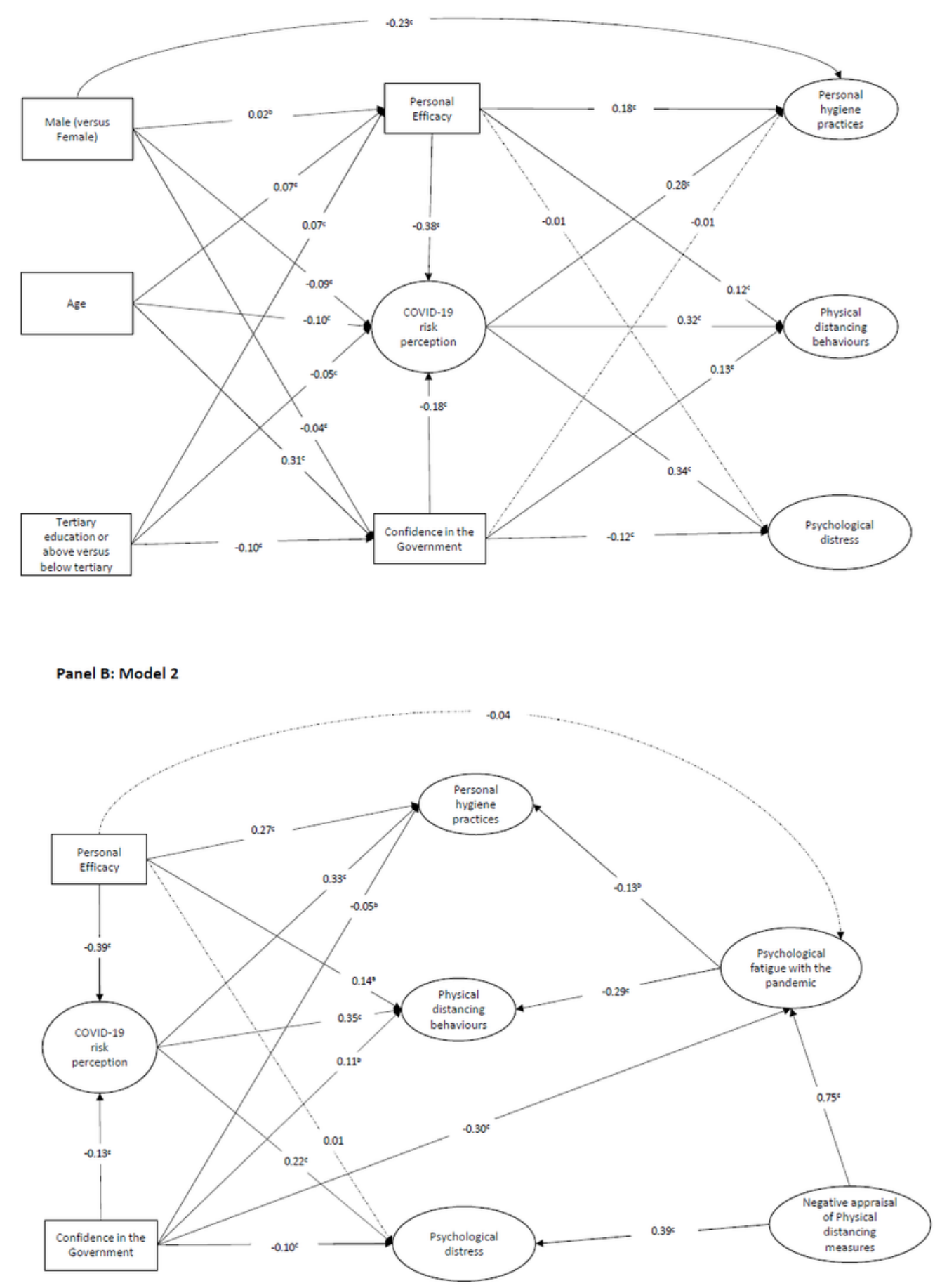

\section{Figure 4}

Associations among stress appraisal, precautionary behaviours and psychological distress and their associated factors based on structural equation modelling (SEM) Variables in Oval shape were treated as latent variables while variables in rectangles were treated in as observed variables in SEM. All numbers on the line arrows were standardized path coefficients. Dotted lines indicate that the path coefficients were not statistically significant. a $p<0.05, b p<0.01, c p<0.001$ 


\section{Supplementary Files}

This is a list of supplementary files associated with this preprint. Click to download.

- Appendixtables.docx

- Supplementaryfiledataset.csv 